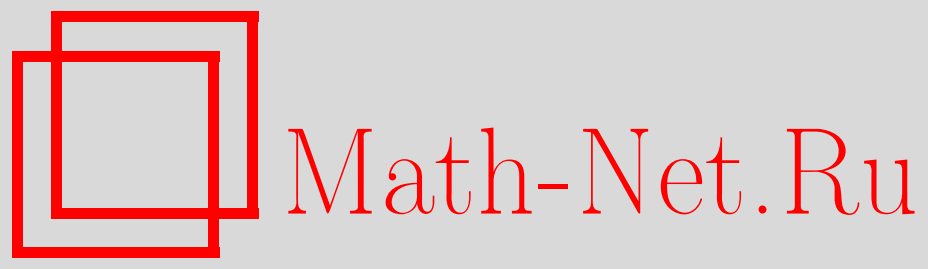

М. М. Маламуд, О полноте системы корневых векторов оператора Штурма-Лиувилля с общими граничными условиями, Функи. анализ и его прил., 2008, том 42, выпуск 3, 45-52

DOI: https://doi.org/10.4213/faa2911

Использование Общероссийского математического портала MathNet.Ru подразумевает, что вы прочитали и согласны с пользовательским соглашением

http://www . mathnet.ru/rus/agreement

Параметры загрузки:

IP : 3.82 .47 .9

26 апреля 2023 г., 16:58:05

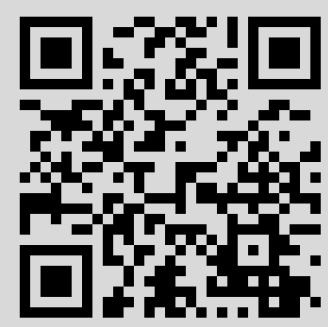


Функииональный анализ и его приложения

2008, т. 42, вып. 3, с. 45-52

УДК 517.43

\title{
О полноте системы корневых векторов оператора Штурма-Лиувилля с общими граничными условиями
}

\author{
(C) 2008. М. М. МАЛАМУД \\ Светлой памяти Марка Григорвевича Крейна посвящается
}

\section{§1. Введение}

Рассмотрим на отрезке $[0,1]$ уравнение Штурма-Лиувилля

$$
-y^{\prime \prime}+q(x) y=\lambda y, \quad q(\cdot) \in L^{1}[0,1],
$$

с краевыми условиями

$$
U_{i}(y)=a_{i 1} y(0)+a_{i 2} y^{\prime}(0)+a_{i 3} y(1)+a_{i 4} y^{\prime}(1)=0, \quad i \in\{1,2\} .
$$

Здесь $q-$ комплекснозначный потенциал, $a_{i k} \in \mathbb{C}$, а граничные формы $U_{1}$ и $U_{2}$ линейно независимы, т. е. матрица $A:=\left(a_{i j}\right)$ имеет ранг 2.

Обозначим через $c(x, \lambda)$ и $s(x, \lambda)$ фундаментальную систему решений уравнения (1.1), выделяемую начальными условиями

$$
c(0, \lambda)=s^{\prime}(0, \lambda)=1, \quad c^{\prime}(0, \lambda)=s(0, \lambda)=0 .
$$

Собственные значения задачи (1.1)-(1.2) являются корнями характеристического определителя $\Delta(\lambda)=\operatorname{det} U(\lambda)$, где

$$
U(\lambda)=\left(\begin{array}{ll}
u_{11}(\lambda) & u_{12}(\lambda) \\
u_{21}(\lambda) & u_{22}(\lambda)
\end{array}\right)=:\left(\begin{array}{ll}
U_{1}(c(x, \lambda)) & U_{1}(s(x, \lambda)) \\
U_{2}(c(x, \lambda)) & U_{2}(s(x, \lambda))
\end{array}\right) .
$$

Раскрывая определитель $\Delta(\lambda)$, находим

$$
\Delta(\lambda)=\mathscr{I}_{12}+\mathscr{I}_{34}+\mathscr{I}_{13} s(1, \lambda)+\mathscr{I}_{14} s^{\prime}(1, \lambda)+\mathscr{I}_{32} c(1, \lambda)+\mathscr{I}_{42} c^{\prime}(1, \lambda),
$$

где $\mathscr{I}_{k p}=\operatorname{det}\left(\begin{array}{ll}a_{1 k} & a_{1 p} \\ a_{2 k} & a_{2 p}\end{array}\right)$ - определитель матрицы из элементов $k$-го и $p$-го столбцов матрицы $A$. При $q=0$ определитель $\Delta_{0}(\lambda)$ задачи (1.1)-(1.2) имеет вид

$$
\Delta_{0}(\lambda)=\left(\mathscr{I}_{12}+\mathscr{I}_{34}\right)+\mathscr{I}_{13} \sin \sqrt{\lambda} / \sqrt{\lambda}+\left(\mathscr{I}_{14}+\mathscr{I}_{32}\right) \cos \sqrt{\lambda}-\mathscr{I}_{42} \sqrt{\lambda} \sin \sqrt{\lambda}
$$

Определение 1. (i) Краевую задачу (1.1)-(1.2) будем называть невырожденной, если $\Delta(\lambda) \neq$ const, т. е. $\left|\mathscr{I}_{13}\right|+\left|\mathscr{I}_{14}\right|+\left|\mathscr{I}_{32}\right|+\left|\mathscr{I}_{42}\right| \neq 0$.

(ii) Краевые условия (1.2) называют невырожденными, если $\Delta_{0}(\lambda) \neq$ const, т. е. $\left|\mathscr{I}_{13}\right|+\left|\mathscr{I}_{42}\right|+\left|\mathscr{I}_{14}+\mathscr{I}_{32}\right| \neq 0$.

Подчеркнем, что невырожденность краевых условий (1.2) не зависит от $q$ и влечет за собой невырожденность краевой задачи (1.1)-(1.2) при любом $q \in$ $L^{1}[0,1]$. Обратное неверно. 
Полнота системы собственных и присоединенных функций (СПФ) задачи (1.1)-(1.2) в случае регулярных (в смысле Биркгофа) краевых условий хорошо известна (см. [8]). Наиболее полный результат о полноте системы СПФ задачи (1.1)-(1.2) получен в [6, теорема 1.3.1].

Теорема 2 [6]. Пусть $q(\cdot) \in L^{1}[0,1]$ и краевые условия (1.2) невырожденны. Тогда система СПФ задачи (1.1)-(1.2) полна в $L^{p}[0,1]$ при $p \in[1, \infty)$.

Отметим, что класс невырожденных краевых условий шире класса регулярных условий (см. замечание 8).

При $q=0$ невырожденность краевых условий (1.2) необходима для полноты системы СПФ. При $q \neq 0$ это уже не так даже в случае $q=\bar{q}$.

В работе изучаются невырожденные краевые задачи (1.1)-(1.2) в случае вырожденных краевых условий $(1.2)$, т. е. в случае $\Delta_{0}(\lambda)=$ const. В этом случае матрица коэффициентов $A$ краевых условий (1.2) имеет вид

$$
A=\left(\begin{array}{llll}
\alpha_{1} & \alpha_{2} & -\alpha \alpha_{1} & \alpha \alpha_{2} \\
\beta_{1} & \beta_{2} & -\alpha \beta_{1} & \alpha \beta_{2}
\end{array}\right), \quad \mathscr{I}_{12}=\operatorname{det}\left(\begin{array}{cc}
\alpha_{1} & \alpha_{2} \\
\beta_{1} & \beta_{2}
\end{array}\right) \neq 0, \quad \alpha \in \mathbb{C} \backslash\{0\} .
$$

Следующая теорема - основной результат работы.

Теорема 3. Пусть краевая задача (1.1)-(1.2) невырожденна и $p \in[1, \infty)$. Если $q(\cdot) \in C^{k}[0,1]$ при некотором $k \in \mathbb{Z}_{+}=\mathbb{N} \cup\{0\}$ u

$$
q^{(k)}(0) \neq(-1)^{k} q^{(k)}(1),
$$

то система СПФ задачи (1.1)-(1.2) полна и минимальна в $L^{p}[0,1]$.

Следствие 4. Пусть краевая задача (1.1)-(1.2) невырожденна и $q(\cdot) \in$ $C[0,1]$. Если $q(0) \neq q(1)$, то система СПФ задачи (1.1)-(1.2) полна и минимальна в $L^{p}[0,1]$ при $p \in[1, \infty)$.

\section{§2. Вспомогательные утверждения}

2.1. Операторы преобразования. Напомним, следуя [3], [6], некоторые факты, касающиеся операторов преобразования, которые существенны в дальнейшем.

Предложение 5 ([3], [6]). Решение $e(x, \lambda)=e\left(x, \mu^{2}\right)$ уравнения (1.1), удовлетворяющее начальным условиям $е(0, \mu)=1, e^{\prime}(0, \mu)=i \mu$, представимо в виде

$$
e\left(x, \mu^{2}\right)=e^{i \mu x}+\int_{-x}^{x} K(x, t) e^{i \mu t} d t
$$

где $K(\cdot, \cdot) \in C(\Omega)$ и $\Omega=\{(x, t): 0 \leqslant|t| \leqslant|x| \leqslant 1\}$. Более того, $K(\cdot, \cdot)$ является (единственным) решением интегрального уравнения

$$
K(x, t)=\frac{1}{2} \int_{0}^{\frac{x+t}{2}} q(s) d s+\int_{0}^{\frac{x+t}{2}} d \alpha \int_{0}^{\frac{x-t}{2}} q(\alpha+\beta) K(\alpha+\beta, \alpha-\beta) d \beta .
$$

При этом если $q(\cdot) \in C^{n}[0,1], n \in \mathbb{Z}_{+}, \operatorname{mo~} K(\cdot, \cdot) \in C^{n+1}(\Omega)$. 
Из равенства (2.1) вытекают следующие представления для решений $c(x, \lambda)$ и $s(x, \lambda)$ при помощи «треугольных» операторов преобразования:

$$
\begin{aligned}
& c\left(x, \mu^{2}\right)=\cos \mu x+\int_{0}^{x} K(x, t ; 0) \cos \mu t d t, \\
& s\left(x, \mu^{2}\right)=\frac{\sin \mu x}{\mu}+\int_{0}^{x} K(x, t ; \infty) \frac{\sin \mu t}{\mu} d t,
\end{aligned}
$$

где $K(x, t, 0):=K(x, t)+K(x,-t)$ и $K(x, t ; \infty):=K(x, t)-K(x,-t)$.

2.2. Основная лемма. Следующая лемма играет ключевую роль в доказательстве теоремы 3. Положим для краткости $q_{I}:=\int_{0}^{1} q(s) d s$ и

$$
r(t):=K_{x}^{\prime}(1, t ; \infty)+K_{t}^{\prime}(1, t ; 0)=K_{x}^{\prime}(1, t)+K_{t}^{\prime}(1, t)-K_{x}^{\prime}(1,-t)-K_{t}^{\prime}(1,-t) .
$$

Лемма 6. Пусть $q(\cdot) \in C^{k}[0,1], k \in \mathbb{Z}_{+} \cdot$ Если $q^{(j)}(0)=(-1)^{j} q^{(j)}(1)$ nри $j \in\{0,1, \ldots, k-1\}$, то справедливо равенство

$$
r^{(k)}(1)=2^{-(k+1)}\left[q^{(k)}(1)-(-1)^{k} q^{(k)}(0)\right] .
$$

Доказательство ведется индукцией по $k$ и в общем случае довольно громоздко. Ограничимся случаями $k=0$ и $k=1$.

(i) Пусть сначала $k=0$. Из уравнения (2.2) вытекают соотношения

$$
K(x, x)=\frac{1}{2} \int_{0}^{x} q(s) d s \quad \text { и } \quad K(x,-x)=0, \quad x \in[0,1] .
$$

Отсюда следует, что

$$
\left.\left(K_{x}^{\prime}+K_{t}^{\prime}\right)\right|_{t=x}=2^{-1} q(x) \quad \text { и }\left.\quad K_{x}^{\prime}(x, t)\right|_{t=-x}=\left.K_{t}^{\prime}(x, t)\right|_{t=-x} .
$$

Дифференцируя уравнение (2.2) по $x$ и $t$ и подставляя в (2.4), получаем

$$
\begin{aligned}
r(t)=\int_{0}^{\frac{1-t}{2}} q\left(\frac{1+t}{2}+\beta\right) K\left(\frac{1+t}{2}+\beta, \frac{1+t}{2}-\beta\right) d \beta+\frac{1}{2}\left[q\left(\frac{1+t}{2}\right)-q\left(\frac{1-t}{2}\right)\right] \\
-\int_{0}^{\frac{1+t}{2}} q\left(\frac{1-t}{2}+\beta\right) K\left(\frac{1-t}{2}+\beta, \frac{1-t}{2}-\beta\right) d \beta .
\end{aligned}
$$

Полагая здесь $t=1$ и учитывая второе из равенств (2.6), приходим к равенству $r(1)=2^{-1}[q(1)-q(0)]$, доказывающему (2.5) при $k=0$.

(ii) Пусть теперь $k=1$ и $q \in C^{1}[0,1]$. Для доказательства равенства (2.5) (при $k=1$ ) докажем более общее тождество

$$
r^{\prime}(1)=4^{-1}\left[q^{\prime}(1)+q^{\prime}(0)\right]+4^{-1}[q(0)-q(1)] q_{I} .
$$

Дифференцируя уравнение (2.2) по $x$, полагая в полученном равенстве $t=-x$ и учитывая второе из соотношений $(2.6)$, получаем

$$
\left.K_{x}^{\prime}(x, t)\right|_{t=-x}=\left.K_{t}^{\prime}(x, t)\right|_{t=-x}=4^{-1} q(0) .
$$


Далее, согласно предложению $5, K(\cdot, \cdot) \in C^{2}(\Omega)$ и в силу $(2.4) r(\cdot) \in$ $C^{1}[0,1]$. Поэтому, дифференцируя $(2.8)$, получаем

$$
\begin{aligned}
r^{\prime}(t)=\frac{1}{4} & {\left[q^{\prime}\left(\frac{1+t}{2}\right)+q^{\prime}\left(\frac{1-t}{2}\right)\right]-\frac{1}{2} q(1)[K(1, t)+K(1,-t)] } \\
& +\int_{0}^{\frac{1-t}{2}} \frac{\partial}{\partial t}\left[q\left(\frac{1+t}{2}+\beta\right) K\left(\frac{1+t}{2}+\beta, \frac{1+t}{2}-\beta\right)\right] d \beta \\
& +\frac{1}{2} \int_{0}^{\frac{1+t}{2}} q^{\prime}\left(\frac{1-t}{2}+\beta\right) K\left(\frac{1-t}{2}+\beta, \frac{1-t}{2}-\beta\right) d \beta \\
& +\frac{1}{2} \int_{0}^{\frac{1+t}{2}} q\left(\frac{1-t}{2}+\beta\right)\left[\left(D_{1}+D_{2}\right) K\left(\frac{1-t}{2}+\beta, \frac{1-t}{2}-\beta\right)\right] d \beta .
\end{aligned}
$$

Полагая в (2.11) $t=1$ и учитывая (2.10) и оба соотношения (2.6), приходим к тождеству (2.9).

Замечание 7. Другой вывод равенства (2.5) можно получить из тождества (5.13) в [4, лемма 11], связывающего ядра двух операторов преобразования.

\section{§3. Доказательство теоремы 3}

(i) Обозначим через $L_{A}$ оператор, порождаемый в $L^{2}[0,1]$ задачей $(1.1)-(1.2)$ с матрицей коэффициентов $A:=\left(a_{i j}\right)$, а через $\sigma\left(L_{A}\right)$ - его спектр. Хорошо известно, что спектр $\sigma\left(L_{A}\right)$ совпадает с совокупностью нулей определителя $\Delta(\cdot)$. При этом кратность $p_{n}$ нуля $\lambda_{n}$ (целой) функции $\Delta(\cdot)$ совпадает с размерностью корневого подпространства

$$
W_{n}:=\operatorname{span}\left\{\operatorname{Ker}\left(L_{A}-\lambda_{n}\right)^{k}: k \in \mathbb{Z}_{+}\right\}, \quad \operatorname{dim} W_{n}=p_{n}
$$

(см. [5], [7], [8]), а геометрическая кратность $m_{n}:=m\left(\lambda_{n}\right):=\operatorname{dim} \operatorname{Ker}\left(L_{A}-\lambda_{n}\right)$ числа $\lambda_{n}$ определяется равенством $m_{n}=2-\operatorname{rank} U\left(\lambda_{n}\right)$.

Рассмотрим решения $w_{i}(x, \lambda):=u_{i 1}(\lambda) s(x, \lambda)-u_{i 2}(\lambda) c(x, \lambda), i \in\{1,2\}$, уравнения (1.1). Ясно, что $U_{i}\left(w_{i}\right)=0, i \in\{1,2\}$, а $U_{1}\left(w_{2}\right)=-U_{2}\left(w_{1}\right)=\Delta(\lambda)$. Поэтому если $m_{n}=1$, то по крайней мере одна из двух систем

$$
\left.D_{\lambda}^{k} w_{i}(x, \lambda)\right|_{\lambda=\lambda_{n}}, \quad k \in\left\{0,1, \ldots, p_{n}-1\right\}, i \in\{1,2\},
$$

образует цепочку (длины $p_{n}$ ) из собственной и присоединенных функций оператора $L_{A}$ (при этом вторая система пропорциональна первой).

Если же $m_{n}=2$, то $U\left(\lambda_{n}\right)=0$ и $w_{1}\left(x, \lambda_{n}\right)=w_{2}\left(x, \lambda_{n}\right)=0$. В этом случае найдутся числа $q_{n i} \in\left\{1, \ldots, p_{n}-1\right\}, i \in\{1,2\}$, такие, что

$$
\left.D_{\lambda}^{k} w_{i}(x, \lambda)\right|_{\lambda=\lambda_{n}}=0, \quad k<q_{n i}, \quad \text { и }\left.\quad D_{\lambda}^{q_{n i}} w_{i}(x, \lambda)\right|_{\lambda=\lambda_{n}} \neq 0, \quad i \in\{1,2\} .
$$

При этом $D_{\lambda}^{q_{n i}} w\left(x, \lambda_{n}\right), i \in\{1,2\}$, являются собственными (возможно, пропорциональными) функциями оператора $L_{A}$, а корневое подпространство попрежнему порождено системой (3.1).

Допуская, что система СПФ (3.1) оператора $L_{A}$ не полна в $L^{2}[0,1]$, найдем ненулевой вектор $f \in L^{2}[0,1]$, ортогональный этой системе. Тогда функции

$$
w_{i}(\lambda ; f):=\int_{0}^{1} w_{i}(x, \lambda) f(x) d x=u_{i 1}(\lambda) s(\lambda ; f)-u_{i 2}(\lambda) c(\lambda ; f), \quad i \in\{1,2\},
$$


являются целыми и в силу свойства (3.2) имеют в каждой точке $\lambda_{n} \in \sigma\left(L_{A}\right)$ нуль кратности $\geqslant p_{n}$. Поэтому функции

$$
\widetilde{w}_{i}(\cdot ; f):=w_{i}(\cdot ; f) / \Delta(\cdot), \quad i \in\{1,2\},
$$

также целые. Оценим их рост, оценив сначала определитель $\Delta(\cdot)$ снизу.

(ii) Пусть условия (1.2) вырожденны, $\Delta_{0}(\lambda)=$ const и для определенности $\mathscr{I}_{12}=1$. В силу $(1.6)$ и (1.7) $\mathscr{I}_{42}=\mathscr{I}_{13}=\mathscr{I}_{14}+\mathscr{I}_{32}=0, \mathscr{I}_{12}+\mathscr{I}_{34}=\left(1-\alpha^{2}\right) \mathscr{I}_{12}$, $\mathscr{I}_{14}=\alpha \mathscr{I}_{12} \neq 0$ и детерминант (1.5) принимает вид

$$
\Delta(\lambda)=b_{0}+\alpha\left[s^{\prime}(1, \lambda)-c(1, \lambda)\right], \quad b_{0}:=1-\alpha^{2} .
$$

Подставляя в (3.4) интегральные представления $(2.3)$ для решений $s(x, \lambda)$ и $c(x, \lambda)$ и интегрируя выражение для $c(1, \lambda)$ по частям, получаем с учетом равенства $K(1,1 ; \infty)=K(1,1 ; 0)$ и определения $(2.4)$

$$
\begin{aligned}
\Delta\left(\mu^{2}\right)=b_{0}+\alpha[K(1,1 & ; \infty) \frac{\sin \mu}{\mu}+\int_{0}^{1} K_{x}^{\prime}(1, t, \infty) \frac{\sin \mu t}{\mu} d t \\
& \left.-\int_{0}^{1} K(1, t ; 0) \cos \mu t d t\right]=b_{0}+\alpha \int_{0}^{1} r(t) \frac{\sin \mu t}{\mu} d t .
\end{aligned}
$$

Представление $(3.5)$, однако, не пригодно для оценки определителя $\Delta(\cdot)$ снизу. Но по условию $q(\cdot) \in C^{k}[0,1]$, и в силу предложения $5 K(\cdot, \cdot) \in C^{k+1}(\Omega)$. Согласно $(2.4), r(\cdot) \in C^{k}[0,1]$. Можно считать, что $q^{(j)}(0)=(-1)^{j} q^{(j)}(1)$ при $j \in\{0,1, \ldots, k-1\}$; в противном случае $k$ следует заменить наименьшим $k_{0}$, для которого выполнено условие (1.8). В силу леммы $6 r^{(j)}(1)=0$ при $j \leqslant k-1$. Учитывая эти равенства, проинтегрируем в (3.5) $k$ раз по частям, считая для определенности, что $k=2 m$. В результате придем к следующему представлению для характеристического определителя:

$$
\Delta\left(\mu^{2}\right)=b_{0}+\sum_{j=0}^{m-1} \frac{b_{j+1}}{\mu^{2 j+2}}+b_{m+1} \frac{\sin \mu}{\mu^{2 m+1}}+(-1)^{m} \int_{0}^{1} r^{(2 m)}(t) \frac{\sin \mu t}{\mu^{2 m+1}} d t,
$$

где $b_{j+1}:=(-1)^{j} \alpha r^{(2 j)}(0), j \in\{0, \ldots, m-1\}$, и $b_{m+1}:=\alpha r^{(2 m-1)}(1)$.

(iii) Представление (3.6) уже пригодно для оценки определителя $\Delta(\cdot)$ снизу, поскольку

$$
b_{m+1}=\alpha r^{(2 m-1)}(1)=\alpha 2^{-2 m}\left[q^{(2 m-1)}(1)+q^{(2 m-1)}(0)\right] \neq 0
$$

(по лемме 6). Так как $|\sin \mu|>C(\rho) \exp (|\operatorname{Im} \mu|)$ вне области $\bigcup_{n} D_{\rho}(n)$, где $D_{\rho}(n)=\{z:|z-n \pi|<\rho\}$ и $\rho<1$, то при каждом $\varepsilon>0$ внутри углов $\Omega_{\varepsilon}^{ \pm}:=\{\mu: \varepsilon \leqslant \pm \arg \mu \leqslant \pi-\varepsilon\}$ справедлива оценка

$$
\left|b_{m+1} \sin \mu+b_{0} \mu^{2 m+1}+\sum_{j=0}^{m-1} b_{j+1} \mu^{2(m-j)-1}\right| \geqslant \frac{\left|b_{m+1}\right|}{2} \exp |\operatorname{Im} \mu|, \quad \mu \in \Omega_{\varepsilon}^{ \pm} .
$$

Кроме того, в силу теоремы Лебега $\lim _{|\mu| \rightarrow \infty} e^{-|\operatorname{Im} \mu|} \int_{0}^{1} r^{(2 m)}(t) \sin \mu t d t=0$. Объединяя эти соотношения с (3.6), приходим к оценке

$$
\left|\Delta\left(\mu^{2}\right)\right| \geqslant C|\mu|^{-2 m-1} \exp |\operatorname{Im} \mu|, \quad \mu \in \Omega_{\varepsilon}^{ \pm} .
$$


С другой стороны, согласно лемме 1.3 .2 из $[6], w_{i}\left(\mu^{2} ; f\right)=o\left(e^{|\operatorname{Im} \mu|}\right),|\mu| \rightarrow \infty$, $i \in\{1,2\}$. Отсюда и из (3.7) вытекает желаемое соотношение

$$
\lim _{r \rightarrow \infty} \max _{|\mu|=r, \mu \in \Omega_{\varepsilon}^{ \pm}}\left|w_{i}\left(\mu^{2} ; f\right) \Delta^{-1}\left(\mu^{2}\right) \mu^{-2 m-1}\right|=0, \quad i \in\{1,2\},
$$

означающее, что $\left|\widetilde{w}_{i}(\mu, f)\right| \leqslant|\mu|^{2 m}$ при $\mu \in \Omega_{\varepsilon}^{ \pm}$. Так как $\widetilde{w}_{i}(\mu, f)-$ функции экспоненциального типа, то из последней оценки на границе $\partial \Omega_{\varepsilon}^{ \pm}$областей $\Omega_{\varepsilon}^{ \pm}$и теоремы Фрагмена-Линделефа вытекает полиномиальная ограниченность функций $\widetilde{w}_{i}$ в дополнительных углах $\mathbb{C} \backslash \Omega_{\varepsilon}^{ \pm}$. По теореме Лиувилля $\widetilde{w}_{i}-$ полиномы степени $\leqslant 2 m$, т. е. $\widetilde{w}_{i}\left(\mu^{2} ; f\right)=P_{i}(\mu), \operatorname{deg} P_{i} \leqslant 2 m$.

(iv) Следовательно, из определения функций $w_{i}(\mu ; f)$ и $\widetilde{w}_{i}(\mu ; f)$ вытекают равенства

$$
\begin{aligned}
& u_{12}(\mu) c(\mu ; f)-u_{11}(\mu) s(\mu ; f)=P_{1}(\mu) \Delta\left(\mu^{2}\right), \\
& u_{22}(\mu) c(\mu ; f)-u_{21}(\mu) s(\mu ; f)=P_{2}(\mu) \Delta\left(\mu^{2}\right) .
\end{aligned}
$$

Домножая первое из них на $u_{22}(\mu)$, а второе - на $u_{12}(\mu)$ и вычитая, получаем после сокращения на $\Delta\left(\mu^{2}\right)$ равенство

$$
s(\mu ; f)=P_{2}(\mu) u_{12}(\mu)-P_{1}(\mu) u_{22}(\mu) .
$$

Далее, в силу леммы Римана-Лебега из (2.3) вытекают соотношения

$$
s(1, \mu)=\mu^{-1}\left[\sin \mu+\varepsilon_{1}(\mu)\right], \quad s^{\prime}(1, \mu)=\cos \mu+\varepsilon_{2}(\mu), \quad \mu \in \mathbb{R},
$$

в которых $\varepsilon_{i}(\mu)=o(1)$ при $\mu=\bar{\mu} \rightarrow \infty, i \in\{1,2\}$. Полагая

$$
Q_{1}:=P_{1} a_{22}-P_{2} a_{12}, \quad Q_{2}:=P_{1} a_{23}-P_{2} a_{13}, \quad Q_{3}:=P_{1} a_{24}-P_{2} a_{14},
$$

а также учитывая (3.11) и равенства

$$
u_{12}(\mu)=a_{12}+a_{13} s(1, \mu)+a_{14} s^{\prime}(1, \mu), \quad u_{22}(\mu)=a_{22}+a_{23} s(1, \mu)+a_{24} s^{\prime}(1, \mu),
$$

запишем (3.10) (при $\mu \in \mathbb{R})$ в виде

$$
s(\mu ; f)=Q_{1}(\mu)+Q_{2}(\mu) \mu^{-1}\left[\sin \mu+\varepsilon_{1}(\mu)\right]+Q_{3}(\mu)\left[\cos \mu+\varepsilon_{2}(\mu)\right] .
$$

Покажем, что (3.13) влечет за собой равенства $Q_{1}=Q_{2}=Q_{3}=0$. Пусть $d:=$ $\max \left\{\operatorname{deg} Q_{j}: 1 \leqslant j \leqslant 3\right\}>0$. Разделив $(3.13)$ на $\mu^{d}$, положив затем $\mu_{k}=\pi(2 k+$ $1 / 2)$ и устремив $k \mathrm{~K} \infty$, получим $\operatorname{deg} Q_{1}<d$. На втором шаге, разделив (3.13) на $\mu^{d}$ и устремив $\mu \mathrm{k} \infty$ по подпоследовательности $\mu_{k}=2 k \pi$, получим $\operatorname{deg} Q_{3}<d$. И наконец, полагая сначала $\mu_{k}=\pi(2 k+1 / 2) \rightarrow \infty$, а затем $\mu_{k}=\pi(2 k-1 / 2)$, разделим обе части равенства (3.13) на $\left(\mu_{k}\right)^{d-1}$ и устремим $k \mathrm{~K} \infty$. Получим $b_{1} \pm b_{2}=0$, где $b_{1}$ и $b_{2}-$ коэффициенты при $\mu^{d-1}$ и $\mu^{d}$ в полиномах $Q_{1}$ и $Q_{2}$ соответственно. Значит, $b_{1}=b_{2}=0$ и $\operatorname{deg} Q_{3}<d$. Полученное противоречие показывает, что $d=0$ и $Q_{j}=C_{j}=$ const. Поэтому равенство (3.13) принимает вид

$$
s(\mu ; f)=C_{1}+C_{2} \mu^{-1}\left[\sin \mu+\varepsilon_{1}(\mu)\right]+C_{3}\left[\cos \mu+\varepsilon_{2}(\mu)\right] .
$$

Полагая в (3.14) $\mu_{k}=\pi(2 k+1 / 2)$, получим при $k \rightarrow \infty$, что $C_{1}=0$. Далее, полагая в (3.14) $\mu_{k}=2 \pi k$ и переходя к пределу при $k \rightarrow \infty$, получаем $C_{3}=0$. И наконец, умножая (3.14) на $\mu$ и учитывая, что $s(\mu ; f)=\mu^{-1} o(1)$, получаем при $\mu_{k}=\pi(2 k+1 / 2) \rightarrow \infty$, что $C_{2}=0$. 
Таким образом, $s(\mu ; f)=0$. Отсюда, используя представление $(2.3)$, легко заключить, что $f=0$ и, значит, система СПФ $\left\{\phi_{j}\right\}_{1}^{\infty}$ оператора $L_{A}$ полна в $L^{2}[0,1]$.

(v) Сопряженный оператор $L_{A}^{*}$ соответствует задаче $(1.1)-(1.2)$ с потенциалом $\bar{q}$ и (некоторой) матрицей $A_{*}$ вместо $q$ и $A$ соответственно. Так как $\bar{q}^{(k)}(0) \neq(-1)^{k} \bar{q}^{(k)}(1)$, то, согласно (iii), система СПФ $\left\{\psi_{j}\right\}_{1}^{\infty}$ оператора $L_{A}^{*}$ полна в $L^{2}[0,1]$. Но система $\left\{\psi_{j}\right\}_{1}^{\infty}$ после надлежащей нормировки биортогональна системе $\left\{\phi_{j}\right\}_{1}^{\infty}$. Значит, система $\left\{\phi_{j}\right\}_{1}^{\infty}$ минимальна в $L^{2}[0,1]$.

Замечание 8. Краевые условия (1.2), регулярные в смысле Биркгофа [8], являются невырожденными. Обратное неверно. Так, например, условия $y^{\prime}(0)+$ $y(1)-y^{\prime}(1)=y(0)+y(1)=0$ и $y^{\prime}(0)+y(1)=y(0)=0$ невырожденны, однако не регулярны по Биркгофу.

\section{§4. Необходимые условия полноты}

Пусть $q \in L^{1}[0,1]$. Положим $\widetilde{q}(x):=q(x)-q(1-x)$.

Предложение 9. Пусть условия (1.2) вырожденны, а краевая задача (1.1)(1.2) - нет. Тогда условие $0 \in \operatorname{supp} \widetilde{q}$ необходимо для полноты в $L^{p}[0,1]$ системы СПФ задачи (1.1)-(1.2). При его нарушении дефект системы СПФ бесконечен.

Доказательство. В данном случае матрица $A$ граничных условий имеет вид (1.7). Так как $\mathscr{I}_{12} \neq 0$, то условия (1.2) эквивалентны следующим:

$$
y(0)=\alpha y(1), \quad y^{\prime}(0)=-\alpha y^{\prime}(1), \quad \alpha \neq 0, \alpha \neq \infty .
$$

Допустим, что $0 \notin \operatorname{supp} \widetilde{q}$. Тогда найдется такое $\varepsilon>0$, что $\widetilde{q}(x)=0$ при п.в. $x \in(0, \varepsilon)$. Поэтому, полагая $z_{i}(x, \lambda):=w_{i}(1-x, \lambda), i \in\{1,2\}$, и учитывая условия (4.1), придем к равенствам (при $i \in\{1,2\}$ )

$$
\left.D_{\lambda}^{k} z_{i}(x, \lambda)\right|_{\lambda=\lambda_{n}}=\left.\alpha^{-1} D_{\lambda}^{k} w_{i}(x, \lambda)\right|_{\lambda=\lambda_{n}}, \quad x \in[0, \varepsilon], k \in\left\{0,1, \ldots, p_{n}-1\right\},
$$

в которых $\lambda_{n}$ - собственное значение задачи (1.1), (4.1), а $p_{n}$ - его алгебраическая кратность. Пусть $f \in L^{2}[0,1], f(x)=0$ при $x \in[\varepsilon, 1-\varepsilon]$ и $f(1-x)=-\alpha f(x)$ при $x \in[0, \varepsilon]$. Тогда в силу (4.2) при $i \in\{1,2\}$ получаем

$$
\begin{array}{r}
\int_{0}^{1} D_{\lambda}^{k} w_{i}\left(x, \lambda_{n}\right) \overline{f(x)} d x=\int_{0}^{\varepsilon} D_{\lambda}^{k} w_{i}\left(x, \lambda_{n}\right) \overline{f(x)} d x+\int_{0}^{\varepsilon} D_{\lambda}^{k} z_{i}\left(x, \lambda_{n}\right) \overline{f(1-x)} d x \\
=\int_{0}^{\varepsilon} D_{\lambda}^{k} w_{i}\left(x, \lambda_{n}\right) \overline{f(x)} d x+\int_{0}^{\varepsilon} \alpha^{-1} D_{\lambda}^{k} w_{i}\left(x, \lambda_{n}\right)(-\alpha) \overline{f(x)} d x=0 .
\end{array}
$$

Учитывая вид (3.1), (3.2) корневых векторов, видим, что система СПФ задачи $(1.1),(4.1)$ не полна и условие $0 \in \operatorname{supp} \widetilde{q}$ необходимо для полноты.

Следствие 10. Пусть $\widetilde{q}(\cdot)$ аналитична в окрестности точки 0. Тогда условие $0 \in \operatorname{supp} \widetilde{q}$ является критерием полноты в $L^{p}[0,1], p \in[1, \infty)$, системы СПФ задачи (1.1)-(1.2).

Доказательство. Пусть $0 \in \operatorname{supp} \widetilde{q}$. Тогда $\widetilde{q}^{(k)}(0)=q^{(k)}(0)-(-1)^{k} q^{(k)}(1) \neq 0$ при некотором $k \in \mathbb{Z}_{+}$в силу аналитичности функции $\widetilde{q}(\cdot)$. По теореме 2 это условие достаточно для полноты системы СПФ задачи. 
Замечание 11. (i) Условие гладкости в теореме 3 можно ослабить до условия $q(\cdot) \in C^{k}([0, \varepsilon) \cup[1-\varepsilon, 1])$.

(ii) Мне не известно, является ли условие $0 \in \operatorname{supp} \widetilde{q}$ достаточным для полноты системы СПФ даже в случае $q \in C^{\infty}[0,1]$. Интересно отметить, что это так в случае задачи Редже (см. [10]).

(iii) В случае $\alpha \neq \pm 1$ условие $\widetilde{q}(x) \equiv 0$ является критерием вольтерровости оператора $L_{A}^{-1}$ (см. [1]). Если же $\widetilde{q}(x) \equiv 0$ и $\alpha= \pm 1$, то $\sigma\left(L_{A}\right)=\mathbb{C}$.

Отметим в заключение работу [9], в которой изучены вопросы полноты и базисности системы СПФ для обыкновенных дифференциальных уравнений $n$-го порядка при различных классах невырожденных краевых условий.

Замечание 12. Теорема 2 гарантирует сохранение полноты при слабом возмущении, а теорема 3 - нет. Так, если $q(0) \neq q(1)$, то оператор $L_{A}(\varepsilon)=-D^{2}+\varepsilon q$ с вырожденными условиями (1.2) является полным при каждом $\varepsilon \neq 0$, а невозмущенный (предельный при $\varepsilon \rightarrow 0$ ) оператор $L_{A}(0)-$ нет. Более того, $L_{A}^{-1}(0)$ вольтерров. Таким образом, теорема 3 дает естественный пример вольтеррова оператора класса $\mathfrak{S}_{2}$, слабые возмущения которого полны. Более того, $L_{A}^{-1}(0)$ является равномерным пределом полных операторов класса $\mathfrak{S}_{2}$.

В заключение я выражаю признательность Л. Л. Оридороге, при обсуждении с которым теоремы 2, принадлежащей В. А. Марченко, мы обратили внимание на отсутствие результатов о полноте системы СПФ задачи (1.1)-(1.2) с вырожденными условиями. Он же сообщил мне, что для задачи (1.1)-(1.2) с $q(x)=x$ и вырожденными условиями (1.2) имеет место полнота. Кроме того, я выражаю признательность М. С. Аграновичу и А. А. Шкаликову за полезные обсуждения, а также благодарность А. А. Шкаликову за информацию о работах [1], [10].

\section{ЛитеРАТУРА}

[1] Б. Н. Бияров, С. А. Джумабаев, Критерий волътерровости краевых задач для уравнения Штурма-Лиувилля, Матем. заметки, 56:1 (1994), 143-146.

[2] И. Ц. Гохберг, М. Г. Крейн, Введение в теорию несамосопряженных операторов, Наука, М., 1965.

[3] Б. М. Левитан, Обратные задачи Штурма-Лиувилля, Наука, М., 1984.

[4] М. М. Маламуд, Подобие операторов Вольтерра и смежные вопросы в теории дифференциальных уравнений дробных порлдков, Труды ММО, 55 (1994), 73-148.

[5] А. С. Маркус, Введение в спектральную теорию полиномиальных операторных пучков, Штиинца, Кишинев, 1986.

[6] В. А. Марченко, Уравнения Штурма-Лиувилля и их приложения, Наукова думка, Киев, 1977.

[7] В. П. Маслов, Операторные методы, Наука, М., 1973.

[8] М. А. Наймарк, Линейнъе дифференциальные операторы, Наука, М., 1969.

[9] А. А. Шкаликов, Краевые задачи для обыкновенных дифференциальных уравнений с параметром в граничных условиях, Труды сем. Петровского, 9 (1983), 190-229.

[10] A. A. Shkalikov, Spectral analysis of the Regge problem, Russ. J. Math. Phys., 8:3 (2001), 356-364.

Институт прикладной математики и механики, Донецк e-mail: mmm@telenet.dn.ua
Поступило в редакцию 14 февраля 2007 г. 